\title{
Investigating Ionic Transport Anisotropy in Oxygen Deficient Lanthanum Cobaltites via STEM and First Principles Theory
}

\author{
Axiel Yaël Birenbaum, ${ }^{1}$ Liang Qiao, ${ }^{2}$ Michael D. Biegalski, ${ }^{3}$ Valentino R. Cooper, ${ }^{1}$ Albina Y. \\ Borisevich $^{1}$ \\ 1. Materials Science and Technology, Oak Ridge National Laboratory, Oak Ridge, TN, USA \\ 2. School of Materials, University of Manchester, Manchester, UK \\ 3. Center for Nanophase Materials Sciences, Oak Ridge National Laboratory, Oak Ridge, TN, USA
}

Oxygen-deficient metal cobalt oxides have been widely studied for solid oxide fuel cell cathode applications. In order to predict atomic-scale transport pathways, a thorough understanding of its defect properties is crucial. Previous Scanning Transmission Electron Microscopy (STEM) studies, demonstrated that lanthanum cobaltite, grown as thin films on [100] $]_{\mathrm{pc}}$ oriented perovskites, exhibit spontaneous ordering of oxygen vacancies [1, 2]. In addition, the magnetic and spin states of cobalt oxides vary dramatically from one case to another case [3, 4].

In this work, we investigate the behavior of $\mathrm{LaCoO}_{3-\delta}$ thin films grown on $\mathrm{SrTiO}_{3}[111]$ using the combination of STEM and density functional theory. For these films, STEM studies reveal ordered vacancy arrangements; lattice spacings computed from STEM images show expansion in the oxygen deficient layers (see Figure 1a). We further use first principles calculations to examine how substrate orientation can be used to shape the anisotropy of oxygen transport. In so doing, we seek to understand the coupling between the structural and electronic properties of $\mathrm{LaCoO}_{3-\delta}$ on $\mathrm{SrTiO}_{3}$, induced by oxygen vacancy ordering.

Two oxygen deficient structures are proposed to fit the experimental observations: $\mathrm{La}_{3} \mathrm{Co}_{3} \mathrm{O}_{8}$ with P21 symmetry, and Brownmilerite $\mathrm{La}_{2} \mathrm{Co}_{2} \mathrm{O}_{5}$. Compared to the bulk $\mathrm{LaCoO}_{3}$ perovskite, $\mathrm{La}_{3} \mathrm{Co}_{3} \mathrm{O}_{8}$ exchanges one oxygen octahedra layer for a tetrahedral layer, and $\mathrm{La}_{2} \mathrm{Co}_{2} \mathrm{O}_{5}$ exchanges two (see Figure 1b). Firstly, we perform a layer-by-layer analysis of the differences between each compositions' structure. This establishes the basis for future study of the dynamics involved going from bulk to $\mathrm{La}_{3} \mathrm{Co}_{3} \mathrm{O}_{8}$ and further to $\mathrm{La}_{2} \mathrm{Co}_{2} \mathrm{O}_{5}$.

Secondly, knowing that cobalitites' electronic structure is known to be particularly sensitive to coordination and structural changes, such as strain or oxygen defects, we address which magnetic and spin states are present in these structures. This leads to an opportunity to discuss the effect of reduced symmetry in oxygen deficient compounds on cobalt oxide behavior compared to the ideal perovskite environment. Finally, we discuss how epitaxial strain leads to oxygen vacancies forming these distinctive stripe patterns. [5].

\section{References:}

[1] Liang Qiao et al., Nano Letters (2015)

[2] Neven Biskup et al., Physical Review Letters (2014)

[3] Bernard Raveau, and Md. Motin Seikh, Handbook of Magnetic (2015)

[4] Yuichi Yamasaki et al., Journal of the Physical Society of Japan (2016)

[5 Research was sponsored by the US Department of Energy (DOE), Ofce of Science, Basic Energy

Sciences (BES), Materials Sciences and Engineering Division This research used resources of 
the National Energy Research Scientic Computing Center, which was supported by the Ofce of Science of the US Department of Energy under Contract No. DE-AC02-05CH11231
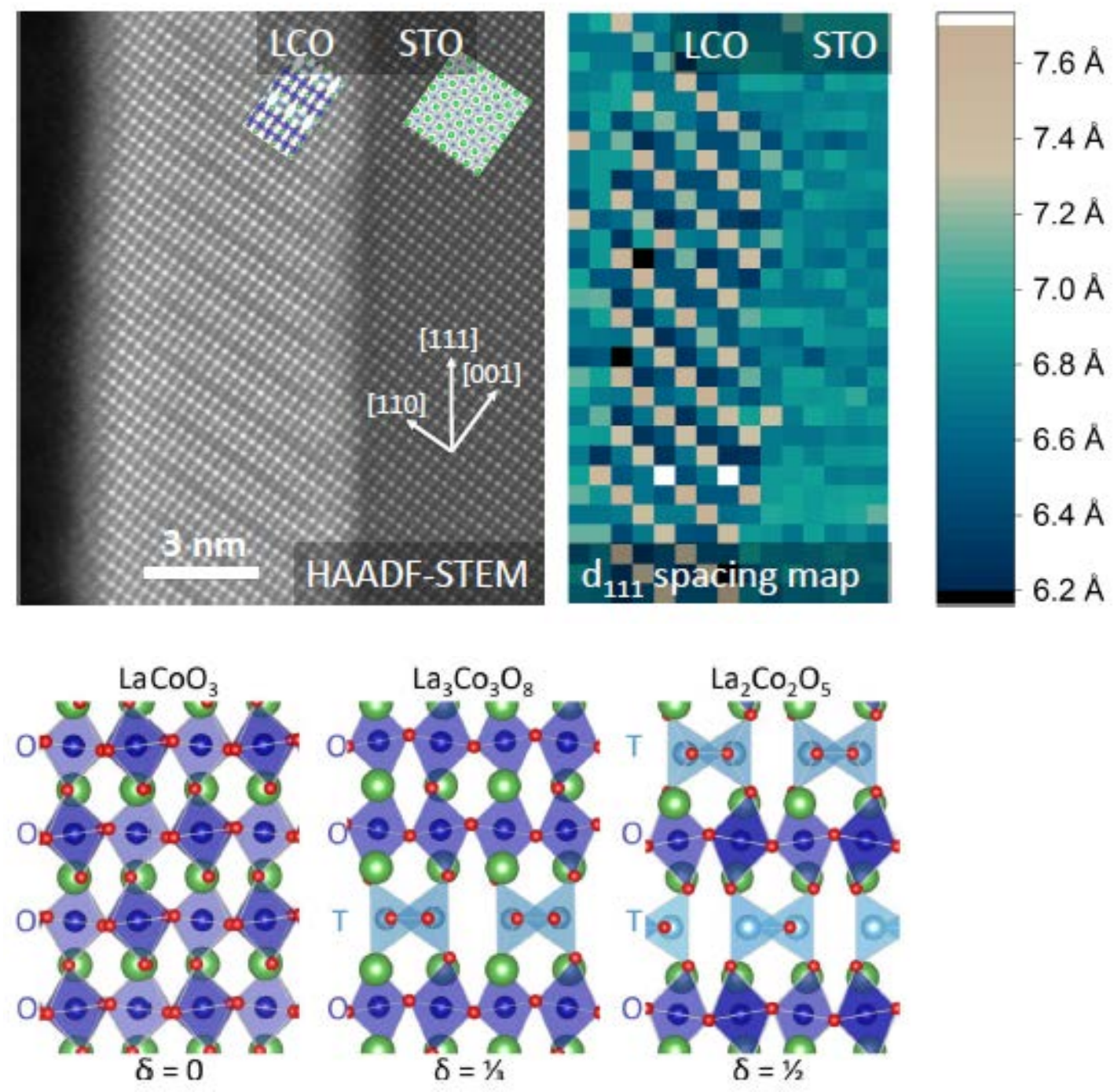

Figure 1. (a) HAADF STEM image and a partial lattice spacing map of $\mathrm{LaCoO}_{3-\delta}$ film on $\mathrm{SrTiO}_{3}$, note 2:1 ordering; (b) $\mathrm{LaCoO}_{3-\delta}$ structures reported in the bulk. 